\title{
Research Article \\ Complete Hausdorffness and Complete Regularity on Supra Topological Spaces
}

\author{
T. M. Al-shami \\ Department of Mathematics, Sana'a University, Sana'a, Yemen \\ Correspondence should be addressed to T. M. Al-shami; tareqalshami83@gmail.com
}

Received 29 January 2021; Revised 3 April 2021; Accepted 29 October 2021; Published 11 November 2021

Academic Editor: Dan Huang

Copyright (C) 2021 T. M. Al-shami. This is an open access article distributed under the Creative Commons Attribution License, which permits unrestricted use, distribution, and reproduction in any medium, provided the original work is properly cited.

\begin{abstract}
The supra topological topic is of great importance in preserving some topological properties under conditions weaker than topology and constructing a suitable framework to describe many real-life problems. Herein, we introduce the version of complete Hausdorffness and complete regularity on supra topological spaces and discuss their fundamental properties. We show the relationships between them with the help of examples. In general, we study them in terms of hereditary and topological properties and prove that they are closed under the finite product space. One of the issues we are interested in is showing the easiness and diversity of constructing examples that satisfy supra $T_{i}$ spaces compared with their counterparts on general topology.
\end{abstract}

\section{Introduction and Preliminaries}

Topological space has been generalized in many manners. They can be classified into three main types; the first one was obtained by strengthening or weakening the conditions of a topology such as Alexendroff topology [1], supra topology [2], and generalized topology [3]. The second one was given by adding newly mathematical structures to the topology such as ordered topology [4], ideal topology [5], and bitopology [6]. The third one was established by defining a topology using one of the generalizations of crisp sets such as fuzzy topology [7] and soft topology [8]. Later on, wide generalizations were constructed by combining two or more types of the previous ones such as fuzzy bitopology [9], ideal ordered bitopology [10], and soft ordered topology [11].

Mashhour et al. [2] introduced the supra topology concept by deleting only the intersection condition. In analogy with topology, they studied the concepts of interior and closure operators, continuity, and separation axioms on supra topology. One of the concrete merits of supra topology is obtaining different examples that give meaning to the concepts and properties defined on a finite set; for example, the only topology defined on a finite set which is a $T_{1}$ space is the discrete topology, whereas there are several sorts of supra topologies that are a $T_{1}$ space. Additionally, it is a hard work to find counterexamples which illustrate that $T_{i-(1 / 2)}$ spaces do not imply $T_{i}$ in the cases of $i=2(1 / 2), 3,3(1 / 2)$, 4 ; see for example, $[12,13]$. After the emergence of supra topology, many authors have explored various topological notions in the spaces of supra topology such as compact $[14,15]$ and paracompact spaces [16], neighborhood system [17], separation axioms [18]), operators, and generalized open sets $[19,20])$. Some published articles demonstrated that many topological findings are still true on supra topologies and illustrated that some of them are invalid like the distributive property of the closure (resp., interior) operator for the union (resp., intersection) between two sets and a compact set in a Hausdorff space is closed. In general, one can note that all topological properties and characterizations which are related to the intersection operator do not remain valid in supra topological spaces. In conclusion, we point to the existence of several studied extensions of supra topology such as supra ordered topology [21], supra fuzzy topology [22], supra soft topology [23], supra ideal topology [24], supra ordered bitopology [25], and supra soft ordered topology [26]. 
In the field of applications, the supra topology represents a framework that is general enough to model phenomena and handle practical issues. In [27], the authors elucidated how supra topological frames induced by semiopen sets handled some digital problems. It is well known that the classes of regular sets and semiopen sets define supra topology structures. In [28], these classes were examined to fix or eliminate obstacles on the digital scope.

Our goal in this article is to complete separation axioms reported in [19] by introducing the concepts of supra completely Hausdorff and supra completely regular spaces. We elaborate their master properties and demonstrate the relationships between them by some illustrative examples. Also, we investigate their behaviours with respect to sub-

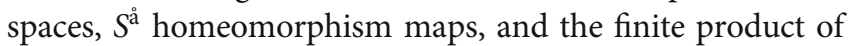
spaces.

Now, we mention some definitions and results in which we need to illustrate the obtained findings.

Definition 1 (see [2]). We call $\Sigma$ as a supra topology on $X$ provided that $\Sigma$ is a subcollection $\Sigma$ of $2^{X}$ such that $X \in \Sigma$ and the arbitrary unions of members of $\Sigma$ are a member of $\Sigma$. A pair $(X, \Sigma)$ is called a supra topological space. Every subset of $X$ that belongs to $\Sigma$ is called supra open, and a set is called supra closed if its complement belongs to $\Sigma$.

To refer supra topological spaces, we use the the pairs $(X, \Sigma)$ and $(Y, \Omega)$.

Definition 2 (see [19]). For a subset $E$ of $(X, \Sigma)$, the union of all supra open sets that is contained in $E$ is called the supra interior of $E$ (denoted by $\operatorname{sint}(E)$ ); the intersection of all supra closed sets that contains $E$ is called the supra closure of $E$ (denoted by $\operatorname{scl}(E)$ ).

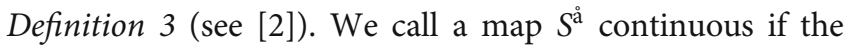
inverse image of every supra open subset is a supra open subset.

Proposition 4 (see [2]). A map from $(X, \Sigma)$ to $(Y, \Omega)$ is an $S^{\mathrm{a}}$ continuous iff $\operatorname{scl}\left(f^{-1}(U)\right) \subseteq f^{-1}(\operatorname{scl}(U))$ for every $U \subseteq Y$.

Definition 5 (see [19]). A supra relative topology on a subset $A$ of $(X, \Sigma)$ is given by $\Sigma_{A}=\{A \bigcap G: G \in \Sigma\}$. A pair $\left(A, \Sigma_{A}\right)$ is said to be a supra subspace of $(X, \Sigma)$.

Lemma 6 (see[19]). A subset $E$ of $\left(A, \Sigma_{A}\right)$, which is a supra subspace of $(X, \Sigma)$, is supra closed iff there is a supra closed subset $F$ of $(X, \Sigma)$ such that $E=A \bigcap F$.

Definition 7 (see $[2,19]) .(X, \Sigma)$ is said to be:

(1) $S T_{0}$ if for any $a \neq b \in X$, there is a supra open set containing (only) one of the points $a$ or $b$

(2) $S T_{1}$ if for any $a \neq b \in X$, there are two supra open sets $U$ and $V$ such that $a \in U / V$ and $b \in U / V$. If $U$ and $V$ are disjoint, then, we call $(X, \Sigma)$ supra Hausdorff (or $\left.S T_{2}\right)$.
(3) Supra regular if for any supra closed set $F$ and every element $a \in F$, there are disjoint supra open sets $U$ and $V$ satisfying that $a \in U$ and $F \subseteq V$

(4) Supra normal if for any disjoint supra closed sets $F$ and $H$, there are disjoint supra open sets $U$ and $V$ satisfying that $F \subseteq U$ and $H \subseteq V$

$(X, \Sigma)$ which is $S T_{1}$ and supra regular (resp., supra normal) is called $S T_{3}$ (resp., $S T_{4}$ ).

Theorem 8 (see [2]). $(X, \Sigma)$ is $S T_{1}$ iff every singleton set is supra closed.

Theorem 9 (see [19]). In $(X, \Sigma)$, the next statements are identical.

(1) $(X, \Sigma)$ is supra regular

(2) For each $a \in X$ and $a \in U \in \Sigma$, there is $V \in \Sigma$ such that $a \in V \subseteq \operatorname{scl}(V) \subseteq U$

(3) Every supra open set $U$ can be written as follows: $U$ $=\bigcup\{H: H \in \Sigma, \operatorname{scl}(H) \subseteq U\}$.

Proposition 10. $(G \times H) \cap(U \times V)=(G \cap H) \times(U \cap V)$.

Definition 11. Let $\left\{\left(X_{i}, \Sigma_{i}\right): i=1,2, \cdots, n\right\}$ be a family of supra topological spaces. We call $\mathscr{B}=\prod_{i=1}^{n} \Sigma_{i}=\left\{\prod_{i=1}^{n}\right.$ $\left.G_{i}: G_{i} \in \Sigma_{i}\right\}$ a base for a supra topology $\Sigma$ on $X=\prod_{i=1}^{n} X_{i}$. A pair $(X, \Sigma)$ is called a finite product supra space.

Proposition 12. Let $A$ and $B$ be subsets of $(X, \Sigma)$ and $(Y, \Omega)$, respectively. Then, $\operatorname{scl}(A) \times \operatorname{scl}(B)=\operatorname{scl}(A \times B)$.

\section{Supra Completely Hausdorff Spaces}

In this portion, we define a concept of supra completely Hausdorff spaces and investigate its master properties. A number of examples which clarify relationships between this concept and some separation axioms that we consider is provided.

Definition 13. $(X, \Sigma)$ is said to be supra completely Hausdorff (or supra $T_{2(1 / 2)}$ ); if for any $a \neq b \in X$, there are two supra open sets $U$ and $V$ containing $a$ and $b$, respectively, such that $\operatorname{scl}(U) \bigcap \operatorname{scl}(V)=\varnothing$.

Proposition 14. Every supra $T_{i}$ space $(X, \Sigma)$ is supra $T_{i-(1 / 2)}$, for $i=3,2(1 / 2)$.

Proof. We prove the proposition in case of $i=3$ and the other case is obvious. Let $a \neq b \in X$. A set $\{b\}$ is supra closed because $(X, \Sigma)$ is supra $T_{1}$. The supra regularity of $(X, \Sigma)$ implies that there are two disjoint supra open sets $U$ and $V$ containing $a$ and $b$, respectively. From Theorem 9, there are supra open sets $G$ and $H$ such that $a \in G \subseteq \operatorname{scl}(G) \subseteq U$ 
and $b \in H \subseteq \operatorname{scl}(H) \subseteq V$. The disjointness of the supra closures of $G$ and $H$ ends the proof.

Corollary 15. When $(X, \Sigma)$ is supra regular, the next concepts are identical.

(1) $(X, \Sigma)$ is supra $T_{0}$

(2) $(X, \Sigma)$ is supra $T_{1}$

(3) $(X, \Sigma)$ is supra $T_{2}$

(4) $(X, \Sigma)$ is supra $T_{2(1 / 2)}$

The converse of the above result is not true in general as the two examples clarify as follows.

Example 16. We construct a supra topology $\Sigma$ on $X=\left\{\omega_{1}\right.$, $\left.\omega_{2}, \omega_{3}, \omega_{4}\right\}$ as follows:

$$
\begin{aligned}
\Sigma= & \left\{\varnothing, X,\left\{\omega_{1}, \omega_{2}\right\},\left\{\omega_{3}, \omega_{4}\right\},\left\{\omega_{1}, \omega_{3}\right\},\left\{\omega_{2}, \omega_{4}\right\},\left\{\omega_{2}, \omega_{3}\right\},\right. \\
& \left.\left\{\omega_{1}, \omega_{2}, \omega_{3}\right\},\left\{\omega_{1}, \omega_{2}, \omega_{4}\right\},\left\{\omega_{1} \omega_{3}, \omega_{4}\right\},\left\{\omega_{2}, \omega_{3}, \omega_{4}\right\}\right\} .
\end{aligned}
$$

It can be checked that $(X, \Sigma)$ is supra $T_{2(1 / 2)}$. On the other hand, $\left\{\omega_{1}, \omega_{4}\right\}$ is a supra closed set and $\omega_{2} \in\left\{\omega_{1}, \omega_{4}\right.$ \} . $(X, \Sigma)$ is not supra $T_{3}$ because there do not exist two disjoint supra open sets such that one of them includes $\omega_{2}$ and the other includes $\left\{\omega_{1}, \omega_{4}\right\}$.

Example 17. We construct a supra topology $\Sigma$ on $X=\{a, b$, $x, y, z\}$ as follows:

$$
\begin{aligned}
\Sigma= & \{\varnothing, X,\{a, b, x, y\},\{a, b, x, z\},\{a, b, y, z\},\{a, x, y, z\}, \\
& \{b, x, y, z\},\{x, y, z\},\{a, b, z\},\{a, x, y\},\{a, b, x\}, \\
& \{a, b, y\},\{b, x, y\},\{a, x\},\{a, y\},\{b, x\},\{b, y\},\{a, b\}, \\
& \{x, y\}\} .
\end{aligned}
$$

Obviously, $(X, \Sigma)$ is supra $T_{2}$. In contrast, $a \neq b$ and the supra closure of any supra open set containing $a$ and the supra closure of any supra open set containing $b$ have a nonempty intersection, so that $(X, \Sigma)$ is not supra $T_{2(1 / 2)}$.

Theorem 18. The two concepts are identical if $|X| \leq 4$ as follows.

(1) $(X, \Sigma)$ is supra $T_{2(1 / 2)}$

(2) $(X, \Sigma)$ is supra $T_{2}$

Proof. The implication $1 \longrightarrow 2$ is obvious.

To prove that $2 \longrightarrow 1$, let $x \neq y \in X$. Then, there are two disjoint supra open subsets $U$ and $V$ of $(X, \Sigma)$ containing $x$ and $y$, respectively. It is well known that $U \cap \operatorname{scl}(V)=\varnothing$ and $\operatorname{scl}(U) \cap V=\varnothing$. Now, we have the following two cases:
(1) $|U|=1$ or $|U|=3$. Then, $U$ is a supra clopen set, so that $\operatorname{scl}(U) \cap \operatorname{scl}(V)=\varnothing$

(2) $|U|=2$. Then, $|V|=2$ or $|V|=1$. If $|V|=2$, then, $U$ and $V$ are supra clopen sets because they are disjoint, so that $\operatorname{scl}(U) \cap \operatorname{scl}(V)=\varnothing$. If $|V|=1$, then, $V$ is a supra clopen set. Thus, $\operatorname{scl}(U) \cap \bar{V}=\varnothing$ $T_{2(1 / 2)}$.

The above two cases end the proof that $(X, \Sigma)$ is supra

Theorem 19. If for every distinct points $a, b$ in $(X, \Sigma)$ there is an $S^{\mathrm{a}}$ continuous function $f$ of $(X, \Sigma)$ into the usual topology $\mathscr{R}$ such that $f(a) \neq f(b)$; then $(X, \Sigma)$ is supra completely Hausdorff.

Proof. Let $a \neq b \in X$. By hypothesis, there exists $x \neq y \in Y$ such that $f(a)=x$ and $f(b)=y$. Since $\mathscr{R}$ is supra completely Hausdorff, there are two disjoint supra open subsets $U$ and $V$ of $\mathscr{R}$ containing $x$ and $y$, respectively, such that $\operatorname{scl}(U)$ $\bigcap \operatorname{scl}(V)=\varnothing$. Since $f$ is an $S^{\text {å }}$ continuous map, $f^{-1}(U)$ and $f^{-1}(V)$ are supra open subsets of $(X, \Sigma)$ containing $a$ and $b$, respectively, such that $f^{-1}(\operatorname{scl}(U)) \bigcap f^{-1}(\operatorname{scl}(V))=$ $\varnothing$. It follows from Proposition 4 that $\operatorname{scl}\left(f^{-1}(U)\right) \subseteq f^{-1}(\mathrm{scl}$ $(U))$ and $\operatorname{scl}\left(f^{-1}(V)\right) \subseteq f^{-1}(\operatorname{scl}(V))$, so that $\operatorname{scl}\left(f^{-1}(U)\right) \bigcap$ $\operatorname{scl}\left(f^{-1}(V)\right)=\varnothing$. Hence, $(X, \Sigma)$ is supra completely Hausdorff, as required.

Definition 20. A map $f:(X, \Sigma) \longrightarrow(Y, \Omega)$ is said to be $S^{a}$ open (resp., $S^{\text {å }}$ closed) if the image of any supra open (resp., supra closed) set is supra open (resp., supra closed). If a bijective map is $S^{a}$ open and $S^{a}$ continuous, then it is called an $S^{a}$ homeomorphism.

Proposition 21. A map $f:(X, \Sigma) \longrightarrow(Y, \Omega)$ is $S^{a ̊}$ closed iff $\operatorname{scl}(f(H)) \subseteq f(\operatorname{scl}(H))$ for every $H \subseteq X$.

Proof. Straightforward.

\section{Definition 22.}

(1) A property that passes from a supra topological space to every supra subspace is called a supra hereditary property

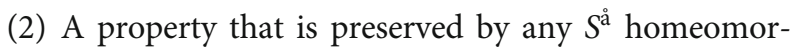
phism map is called a supra topological property

Theorem 23. Let $f:(X, \Sigma) \longrightarrow(Y, \Omega)$ be a bijective $S^{a}$ open map. If $(X, \Sigma)$ is supra completely Hausdorf, then, $(Y, \Omega)$ is supra completely Hausdorff.

Proof. Let $x \neq y \in Y$. Then, it follows from the bijective of $f$ that there are $a \neq b \in X$ such that $a=f^{-1}(x)$ and $b=f^{-1}(y)$. Since $(X, \Sigma)$ is supra completely Hausdorff, there are two disjoint supra open subsets $U$ and $V$ of $(X, \Sigma)$ containing $a$ and $b$, respectively, such that $\operatorname{scl}(U) \bigcap \operatorname{scl}(V)=\varnothing$. Now, 
$f(U)$ and $f(V)$ are supra open subsets of $(Y, \Omega)$ containing $x$ and $y$, respectively, such that $f(\operatorname{scl}(U)) \bigcap f(\operatorname{scl}(V))=\varnothing$. Since $f$ is a bijective $S^{\text {a }}$ open map, it is also an $S^{\text {a }}$ closed map, so that $\operatorname{scl}(f(U)) \subseteq f(\operatorname{scl}(U))$ and $\operatorname{scl}(f(V)) \subseteq f(\operatorname{scl}(V))$. Thus, $\operatorname{scl}(f(U)) \bigcap \operatorname{scl}(f(V))=\varnothing$. Hence, $(Y, \Omega)$ is a supra completely Hausdorff, as required.

Corollary 24. The property of being a supra completely Hausdorff space is a supra topological property.

Lemma 25. Consider that $\left(A, \Sigma_{A}\right)$ is a subspace of $(X, \Sigma)$. Then, $\quad(\operatorname{scl}(U))_{A}=A \bigcap \operatorname{scl}(U)$ for each $U \subseteq A$, where $(\operatorname{scl}(U))_{A}$ is the supra closure operator in $\left(A, \Sigma_{A}\right)$.

Proof. $(\operatorname{scl}(U))_{A}=\cap\left\{H: U \subseteq H\right.$ and $\left.A / H \in \Sigma_{A}\right\}=\cap\{A \cap F$ $: U \subseteq A \cap F$ and $\left.F^{c} \in \Sigma\right\}=\cap\left\{A \cap F: U \subseteq F\right.$ and $\left.F^{c} \in \Sigma\right\}=A$ $\cap\left[\cap\left\{F: U \subseteq F\right.\right.$ and $\left.\left.F^{c} \in \Sigma\right\}\right]=A \cap \operatorname{scl}(U)$

Theorem 26. The property of being a supra completely Hausdorff space is a supra hereditary property.

Proof. Suppose that $\left(E, \Sigma_{E}\right)$ is a subspace of a supra completely Hausdorff space $(X, \Sigma)$. Let $x \neq y \in E \subseteq X$. Then, there are two disjoint supra open subsets $U$ and $V$ of $(X, \Sigma)$ containing $x$ and $y$, respectively, such that $\operatorname{scl}(U) \cap \operatorname{scl}(V)$ $=\varnothing$. Now, $G=E \cap U$ and $H=E \cap V$ are two disjoint supra open subsets of $\left(E, \Sigma_{E}\right)$ containing $x$ and $y$, respectively. From Lemma 25, we obtain $(\operatorname{scl}(G))_{E}=E \cap \operatorname{scl}(G)=E \cap \mathrm{scl}$ $(U \cap E) \subseteq E \cap \operatorname{scl}(U)$. Similarly, $(\operatorname{scl}(H))_{E} \subseteq E \cap \operatorname{scl}(V)$. The disjointness of $(\operatorname{scl}(G))_{E}$ and $(\operatorname{scl}(H))_{E}$ shows that $\left(E, \Sigma_{E}\right)$ is a supra completely Hausdorff.

Theorem 27. The finite product of supra completely Hausdorff spaces is a supra completely Hausdorff.

Proof. Consider $(X, \Sigma)$ and $(Y, \Omega)$ as two supra topological spaces.

Suppose that $\left(x_{1}, y_{1}\right) \neq\left(x_{2}, y_{2}\right)$. This means that either $x_{1} \neq x_{2}$ or $y_{1} \neq y_{2}$. Say, $x_{1} \neq x_{2}$. Therefore, there are two disjoint supra open subsets $U$ and $V$ of $(X, \Sigma)$ containing $x_{1}$ and $x_{2}$, respectively, such that $\operatorname{scl}(U) \bigcap \operatorname{scl}(V)=\varnothing$. Now, $U \times Y$ and $V \times Y$ are two disjoint supra open subsets of $(X$ $\times Y, \Sigma \times \Omega)$ containing $\left(x_{1}, y_{1}\right)$ and $\left(x_{2}, y_{2}\right)$, respectively. It follows from Proposition 12 and Proposition 10 that $\operatorname{scl}(U$ $\times Y) \bigcap \operatorname{scl}(V \times Y)=\varnothing$. Hence, $(X \times Y, \Sigma \times \Omega)$ is a supra completely Hausdorff.

\section{Supra Completely Regular Spaces}

In this portion, we first investigate some properties of supra regular and supra normal spaces. Then, we define a concept of supra completely regular spaces. Compared with completely regular on topological spaces, the continuous functions are replaced by $S^{\mathfrak{a}}$ continuous functions; however, the codomain remains $[0,1]$ which is a subspace of the usual topology. We discuss some rudiments of a supra completely regular space with the help of examples and characterize it in many ways.
Theorem 28. If $|X| \leq 4$, then, every supra regular space ( $X$, $\Sigma)$ is supra normal.

Proof. Let $F$ and $H$ be two disjoint nonempty supra closed subsets of a supra regular space $(X, \Sigma)$. Now, we have the following four cases:

(1) $|F|=4$. Then, $H$ is the empty set. Hence, the proof is trivial

(2) $|F|=3$. Since $F$ and $H$ are disjoint, it must be that $H$ is a singleton, so that $F$ and $H$ are disjoint supra open sets as well

(3) $|F|=2$. Then, $|H|=1$ or $|H|=2$. If $|H|=1$, say $H=\{x\}$. Then, $x \in F$. It follows by the regularity of $(X, \Sigma)$ that there are two disjoint supra open subsets $U$ and $V$ of $(X, \Sigma)$ containing $F$ and $H$, respectively. If $|H|=2$, then, $F$ and $H$ are disjoint supra open sets as well

(4) $|F|=1$, say $F=\{x\}$. Then, $x \in H$. It follows by the regularity of $(X, \Sigma)$ that there are two disjoint supra open subsets $U$ and $V$ of $(X, \Sigma)$ containing $F$ and $H$, respectively

(5) $|F|=0$. Then, $\varnothing$ and $X$ are two disjoint supra open subsets of $(X, \Sigma)$ containing $F$ and $H$, respectively

The above five cases end the proof that $(X, \Sigma)$ is supra normal.

Corollary 29. The next two concepts are identical if $|X| \leq 4$.

(1) $(X, \Sigma)$ is supra $T_{4}$

(2) $(X, \Sigma)$ is supra $T_{3}$

By the next examples, we illustrate that the converse of Theorem 28 fails and elucidate that the concepts of supra normal and supra regular spaces are independent of each other when $|X|>4$.

Example 30. Consider that $\Sigma=\{\varnothing, X,\{a\},\{a, b\},\{b, c\}\}$ is a supra topology on $X=\{a, b, c\}$. Obviously, $(X, \Sigma)$ is supra normal. In contrast, $b \in\{c\}$ and $\{c\}$ is a supra closed set. $(X, \Sigma)$ is not supra regular because there do not exist two disjoint supra open sets separate $b$ and $\{c\}$.

Example 31. Consider that $\Sigma=P(X) \backslash\{\{a\},\{x\},\{a, b\},\{x$, $y\}\}$ is a supra topology on $X=\{a, b, x, y, z\}$. Now, $\{a, b\}$ and $\{x, y\}$ are supra closed subsets of $(X, \Sigma)$. One can check that $(X, \Sigma)$ is supra regular. In contrast, $(X, \Sigma)$ is not supra normal because there do not exist two disjoint supra open sets separate $\{a, b\}$ and $\{x, y\}$.

The following result is the version of Urysohn's lemma on supra topology and has a similar proof to Urysohn's lemma on general topology, so that the proof is omitted. 
Theorem 32. $(X, \Sigma)$ is supra normal iff for each disjoint supra-closed sets $A, B$ there exists an $S^{\mathrm{a}}$ continuous map $g$ $: X \longrightarrow[0,1]$ such that $g(a)=0$ for each $a \in A$ and $g(b)=1$ for each $b \in B$.

Take into consideration that a supra topology which is defined on $[0,1]$ is a subspace of the usual topological space $(\mathscr{R}, \Sigma)$.

Definition 33. $(X, \Sigma)$ is said to be

(1) Supra completely regular if for each $x \in X$ and supra closed set $F$ such that if $x \in F$, there exists an $S^{a}$ continuous map $g: X \longrightarrow[0,1]$ such that $g(x)=0$ and $g(F)=1$

(2) Supra $T_{3(1 / 2)}$ if it is supra completely regular and supra $T_{1}$

Proposition 34. The following three statements are identical:

(1) $(X, \Sigma)$ is supra completely regular

(2) For each $x \in X$ and supra closed set $F$ such that $x \in F$, there exists an $S^{\mathrm{a}}$ continuous map $\mathrm{g}: X \longrightarrow[0,1]$ such that $g(x)=1$ and $g(F)=0$

(3) For each $x \in X$ and supra open set $U$ containing $x$, there exists an $S^{\mathrm{a}}$ continuous map $g: X \longrightarrow[0,1]$ such that $g(x)=1$ and $g\left(U^{c}\right)=0$

Proof. $1 \longrightarrow 2$. Since $(X, \Sigma)$ is supra completely regular, then for each $x \in X$ and supra closed set $F$ such that $x \in F$, there

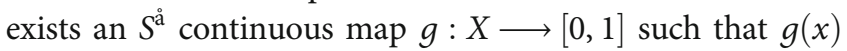
$=0$ and $g(F)=1$. Therefore, a map $h(x)=1-g(x)$ of $(X$, $\Sigma)$ into $[0,1]$ is $S^{a}$ continuous. Hence, $h(x)=1$ and $h(F)=$ 0 , as required.

$2 \longrightarrow 3$, obviously.

$3 \longrightarrow 1$. Let $F$ be a supra closed set and $x \in X$ such that $x \in F$. Then, $F^{c}$ is a supra open set. By hypothesis, there exists an $S^{\mathfrak{a}}$ continuous map $g: X \longrightarrow[0,1]$ such that $g(x)$ $=1$ and $g\left(\left(F^{c}\right)^{c}\right)=g(F)=0$. Therefore, a map $h(x)=1-g$ $(x)$ of $(X, \Sigma)$ into $[0,1]$ is $S^{a}$ continuous. Since $h(x)=0$ and $h(F)=1$, then, $(X, \Sigma)$ is supra completely regular.

Theorem 35. For every distinct points $a, b$ in a supra $T_{3(1 / 2)}$ space $(X, \Sigma)$, there is an $S^{\AA}$ continuous function $f:(X, \Sigma)$ $\longrightarrow \mathscr{R}$ such that $f(a) \neq f(b)$.

Proof. Let $a \neq b \in X$. Then, $\{b\}$ is a supra closed set because $(X, \Sigma)$ is supra $T_{1}$. Since $a \in\{b\}$, it follows from the supra complete regularity of $(X, \Sigma)$ that there is an $S^{a}$ continuous map $g:(X, \Sigma) \longrightarrow[0,1]$ such that $g(a)=0$ and $g(b)=1$. Hence, $g(a) \neq g(b)$.

Definition 36. For an $S^{\mathfrak{a}}$ continuous map $g$ of $X$ into $\mathscr{R}$, we define
(1) $g^{+}$of $X$ into $\mathscr{R}$ by $g^{+}(x)=\sup \{g(x), 0\}$. It is obvious that $g^{+}$is $S^{\text {a }}$ continuous

(2) The zero set $Z(g)$ by $Z(g)=\{x \in X: g(x)=0\}$

Theorem 37. $(X, \Sigma)$ is supra completely regular iff for every $x \in X$ and supra closed set $F$ such that $x \in F$, there are $S^{\mathrm{a}}$ continuous maps $f$ and $g$ of $(X, \Sigma)$ into $\mathscr{R}$ such that $x \in$ $\sin t[Z(f)], F \subseteq \sin t[Z(g)]$, and $Z(f) \cap Z(g)=\varnothing$.

Proof.

(1) Necessity. Suppose that $F$ is a supra closed set and $x \in X$ such that $x \in F$. Then, there is an $S^{\mathfrak{a}}$ continuous map $h:(X, \Sigma) \longrightarrow \mathscr{R}$ such that $h(x)=0$ and $h(F)$ $=1$. Let $f(x)=(h-(2 / 5))^{+}$and $g(x)=((3 / 5)-h)^{+}$. Obviously, the two sets $Z(f)$ and $Z(g)$ are disjoint. Now, $x \in h^{-1}(-\infty,(2 / 5)) \subseteq Z(f)$ and $F \subseteq h^{-1}((3 / 5)$, $\infty) \subseteq Z(g)$. By the $S^{\text {a }}$ continuity of $h$, it follows that $h^{-1}(-\infty,(2 / 5))$ and $h^{-1}((3 / 5), \infty)$ are supra open subsets of $(X, \Sigma)$. Hence, the necessary part is proved

(2) Sufficiency. Let $F$ be a supra closed set and $x \in X$ such that $x \in F$. Take two maps $f$ and $g$ as described in the theorem. Then, we have $f^{2}(x)+g^{2}(x) \neq 0$ for each $x \in X$. Therefore, $h(x)=f^{2}(x)\left[f^{2}(x)+g^{2}(x)\right]^{-1}$ is an $S^{\mathrm{a}}$ continuous map such that $h(x)=0$ and $h(F)$ $=1$. Hence, $(X, \Sigma)$ is supra completely regular

Theorem 38. Every supra completely regular space $(X, \Sigma)$ is supra regular.

Proof. Let $F$ be a supra closed subset of $(X, \Sigma)$ and let $x \in F$. Since $(X, \Sigma)$ is supra completely regular, there is an $S^{\mathfrak{a}}$ continuous map $g: X \longrightarrow[0,1]$ such that $g(x)=0$ and $g(F)$ $=1$. Since $[0,1]$ is a subspace of the usual topological space $(\mathscr{R}, \Sigma)$, it is supra $T_{2}$. So that there are two disjoint supra open sets $U$ and $V$ containing 0 and 1 , respectively. It follows from the $S^{\text {a }}$ continuity of $g$ that $g^{-1}(U)$ and $g^{-1}(V)$ are two disjoint supra open sets containing $x$ and $F$, respectively. Hence, $(X, \Sigma)$ is supra regular.

Corollary 39. Every supra $T_{3(1 / 2)}$ space $(X, \Sigma)$ is supra $T_{3}$.

Theorem 40. A supra normal space $(X, \Sigma)$ is supra completely regular iff it is supra regular.

Proof.

(1) Necessity. It follows from the above theorem

(2) Sufficiency. Let $F$ be a supra closed subset of $(X, \Sigma)$ and let $x \in F$. By hypothesis, there are two disjoint supra open sets $U$ and $V$ such that $x \in U$ and $F \subseteq$ $V$. Now, $F$ and $V^{c}$ are two disjoint supra closed sets. By using Theorem 32, we can find an $S^{\text {a }}$ continuous 
map $g: X \longrightarrow[0,1]$ such that $g(a)=0$ for each $a$ $\in V^{c}$ and $g(b)=1$ for each $b \in F$. This means that $g(x)=0$ and $g(b)=1$ for each $b \in F$. Hence, $(X, \Sigma)$ is supra completely regular.

Corollary 41. Every supra $T_{4}$ space $(X, \Sigma)$ is supra $T_{3(1 / 2)}$.

Lemma 42. The restricted map of an $S^{a ̊}$ continuous map is $S^{\mathrm{a}}$ continuous.

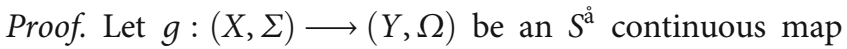
and let $A \subseteq X$. To prove that $g \mid A:\left(A, \Sigma_{A}\right) \longrightarrow(Y, \Omega)$ is $S^{\text {å }}$ continuous, let $G$ be a supra open subset of $Y$. Then, $g^{-1}(G)$ is a supra open subset of $(X, \Sigma)$. Now, $A \cap g^{-1}(G)$ is a supra open subset of $\left(A, \Sigma_{A}\right)$. Thus, $g \mid A$ is $S^{\text {a }}$ continuous.

Theorem 43. The property of being a supra completely regular space is a supra hereditary property.

Proof. Consider that $\left(A, \Sigma_{A}\right)$ is a supra subspace of a supra completely regular space $(X, \Sigma)$. Let $E$ be a supra closed subset of $\left(A, \Sigma_{A}\right)$ and $x \in A$ such that $x \in E$. Then, it follows from Lemma 6 that there is a supra closed subset $F$ of $(X$, $\Sigma)$ such that $E=A \bigcap F$. Obviously, $x \in F$. By the supra complete regularity of $(X, \Sigma)$, there is an $S^{\text {å }}$ continuous map $g: X \longrightarrow[0,1]$ such that $g(x)=0$ and $g(F)=1$. Thus, $g \mid A:\left(A, \Sigma_{A}\right) \longrightarrow[0,1]$ is an $S^{\text {a }}$ continuous map such that $g \mid A(x)=0$ and $g|A(E)=g| A(A \cap F)=1$. This ends the proof that a supra completely regular space is a supra hereditary property.

Corollary 44. A supra $T_{3(1 / 2)}$ space is a supra hereditary property.

Theorem 45. A supra completely regular space is a supra topological property.

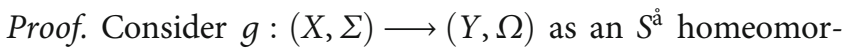
phism and let $(X, \Sigma)$ be a supra completely regular space. Suppose that $F$ is a supra closed subset of $(Y, \Omega)$ and $y \in Y$ such that $y \in F$. Then, $g^{-1}(F)$ is a supra closed subset of $(X, \Sigma)$ and $g^{-1}(y) \in g^{-1}(F)$. Since $(X, \Sigma)$ is supra completely regular, there is an $S^{\text {a }}$ continuous map $h: X \longrightarrow[0,1]$ such that $h\left(g^{-1}(y)\right)=0$ and $h\left(g^{-1}(F)\right)=1$. Since $g$ is an $S^{\text {a homeo- }}$ morphism, then, $g^{-1}$ is $S^{\mathrm{a}}$ continuous. Therefore, $p=h \circ g^{-1}$ $:(Y, \Omega) \longrightarrow[0,1]$ is $S^{\text {a }}$ continuous such that $p(y)=\left(h \circ g^{-1}\right)$ $(y)=h\left(g^{-1}(y)\right)=0$ and $p(F)=\left(h \circ g^{-1}\right)(F)=h\left(g^{-1}(F)\right)=1$. Hence, $(Y, \Omega)$ is a supra completely regular space.

Corollary 46. A supra $T_{3(1 / 2)}$ space is a supra topological property.

Theorem 47. The finite product of supra completely regular spaces is supra completely regular.
Proof. Let $(X \times Y, \Sigma \times \Omega)$ be the product spaces of two supra completely regular spaces $(X, \Sigma)$ and $(Y, \Omega)$. Suppose that $(x, y) \in X \times Y$ and $G=\bigcup_{i \in I}\left(U_{i} \times V_{i}\right)$ be a supra open subset of $X \times Y$ such that $(x, y) \in G$. Now, there exists $\alpha \in I$ such that $(x, y) \in U_{\alpha} \times V_{\alpha}$. Then, for each $x \in X$ and supra open set $U_{\alpha}$ containing $x$, there exists an $S^{\text {a }}$ continuous map $g_{1}$ $: X \longrightarrow[0,1]$ such that $g_{1}(x)=1$ and $g_{1}\left(U_{\alpha}^{c}\right)=0$, and for each $y \in Y$ and supra open set $V_{\alpha}$ containing $y$, there exists an $S^{\text {å }}$ continuous map $g_{2}: X \longrightarrow[0,1]$ such that $g_{2}(y)=1$ and $g_{2}\left(V_{\alpha}^{c}\right)=0$. Now, we define a map $f: X \times Y \longrightarrow[0,1]$ by $f(u, v)=g_{1}(u) \times g_{2}(v)$. It can be checked that $f$ is $S^{a}$ continuous, we have $f(x, y)=g_{1}(x) \times g_{2}(y)=1 \times 1=1$ and $f\left(\left(U_{\alpha} \times V_{\alpha}\right)^{c}\right)=f\left[\left(U_{\alpha}^{c} \times Y\right) \bigcup\left(Y \times V_{\alpha}^{c}\right)\right]=f\left[\left(U_{\alpha}^{c} \times Y\right)\right] \bigcup f[$ $\left.\left(Y \times V_{\alpha}^{c}\right)\right]=g_{1}\left(U_{\alpha}^{c} \times Y\right) \bigcup g_{2}\left(Y \times V_{\alpha}^{c}\right)=\left[g_{1}\left(U_{\alpha}^{c}\right) \times g_{1}(Y)\right]$ $\bigcup\left[g_{2}(Y) \times g_{2}\left(V_{\alpha}^{c}\right)\right]=\left[0 \times g_{1}(Y)\right] \bigcup\left[g_{2}(Y) \times 0\right]=\{0\}$. Since $\left[\bigcup_{i \in I}\left(U_{i} \times V_{i}\right)\right]^{c} \subseteq\left(U_{\alpha} \times V_{\alpha}\right)^{c}$, then, $f\left(\left[\bigcup_{i \in I}\left(U_{i} \times V_{i}\right)\right]^{c}\right)=0$. Hence, $(X \times Y, \Sigma \times \Omega)$ is supra completely regular.

Corollary 48. The property of being a supra $T_{3(1 / 2)}$ space is preserved under a finite product.

\section{Conclusion}

In this paper, we have highlighted two concepts on supra topological spaces, namely, supra completely Hausdorff and supra completely regular spaces. We have investigated some of their characterizations and provided some examples to show the relationships between them. Also, we have proved that they are hereditary and topological properties as well as they are closed under the finite product of spaces.

In future works, we plan to study an application of supra topology on the information system and apply the concepts given herein and those in [19] to categorize the approximation spaces. Also, we will study functionally separation axioms [29] in the frame of supra topologies and elaborate their relationships with the concepts presented herein.

\section{Data Availability}

No data were used to support this study.

\section{Conflicts of Interest}

The author declares that he has no competing interests.

\section{References}

[1] A. D. Alexendroff, "Additive set functions in abstract spaces," Recueil Mathématique (Nouvelle série), vol. 8, no. 50, pp. 307348, 1940.

[2] A. S. Mashhour, A. A. Allam, F. S. Mahmoud, and F. H. Kheder, "On supra topological spaces," Indian Journal of Pure and Applied Mathematics, vol. 14, no. 4, pp. 502-510, 1983.

[3] Á. Császár, "Generalized topology, generized continuity," Acta Mathematica Hungarica, vol. 96, no. 4, pp. 351-357, 2002.

[4] L. Nachbin, Topology and Order, D. Van Nostrand Inc., Princeton, New Jersey, 1965.

[5] K. Kuratowski, Topology, Vol. I, Academic Press, New York, 1966. 
[6] J. C. Kelly, "Bitopological spaces," Proceedings of the London Mathematical Society, vol. s3-13, no. 1, pp. 71-89, 1963.

[7] C. L. Chang, "Fuzzy topological spaces," Journal of Mathematical Analysis and Applications, vol. 24, no. 1, pp. 182-190, 1968.

[8] M. Shabir and M. Naz, "On soft topological spaces," Computers and Mathematics with Applications, vol. 61, no. 7, pp. 1786-1799, 2011.

[9] A. Kandil and M. El-Shafee, "Separation axioms for fuzzy bitopologicalspaces," Journal of Institute of Mathematics and Computer Sciences, vol. 4, pp. 373-383, 1991.

[10] A. Kandil, O. Tantawy, S. A. El-Sheikh, and M. Hosny, "New approach to bitopological ordered spaces," Jökull Journal, vol. 63, no. 6, pp. 10-25, 2013.

[11] T. M. Al-shami, M. E. El-Shafei, and M. Abo-Elhamayel, "On soft topological ordered spaces," Journal of King Saud University-Science, vol. 31, no. 4, pp. 556-566, 2019.

[12] A. Mysior, "A regular space which is not completely regular," Proceedings of the American Mathematical Society, vol. 81, p. 652,1981

[13] A. B. Raha, "An example of a regular space that is not completely regular," Proceedings Mathematical Sciences, vol. 102, no. 1, pp. 49-51, 1992.

[14] T. M. Al-shami, "Supra semi-compactness via supra topological spaces," Journal of Taibah University for Science, vol. 12, no. 3, pp. 338-343, 2018.

[15] J. M. Mustafa, "Supra b-compact and supra b-Lindelöf spaces," Journal of Mathematics and Applications, vol. 36, pp. 79-83, 2013.

[16] T. M. Al-shami, "Paracompactness on supra topological spaces," Journal of Linear and Topological Algebra, vol. 9, no. 2, pp. 1-7, 2020.

[17] Y. K. Kim and W. K. Min, "Some results on supraneighborhood spaces," International Journal of Mathematics Analysis, vol. 8, pp. 2311-2317, 2014.

[18] M. E. El-Shafei, A. H. Zakari, and T. M. Al-shami, "Some applications of supra preopen sets," Journal of Mathematics, vol. 2020, Article ID 9634206, 11 pages, 2020.

[19] T. M. Al-shami, "Some results related to supra topological spaces," Journal of Advanced Studies in Topology, vol. 7, no. 4, pp. 283-294, 2016.

[20] O. R. Sayed and T. Noiri, "On supra b-open sets and supra bcontinuity on topological spaces," European Journal of pure and applied Mathematics, vol. 3, pp. 295-302, 2010.

[21] P. Das, "Separation axioms in ordered spaces," Soochow Journal of Mathematics, vol. 30, no. 4, pp. 447-454, 2004.

[22] M. E. A. EL-Monsef and A. E. Ramadan, "On fuzzy supra topological spaces," Indian Journal of Pure and Applied Mathematics, vol. 18, no. 4, pp. 322-329, 1987.

[23] S. A. el-Sheikh and A. M. Abd el-latif, "Decompositions of some types of supra soft sets and soft continuity," International Journal of Mathematics Trends and Technology, vol. 9, no. 1, pp. 37-56, 2014.

[24] S. Modak and S. Mistry, "Ideal on supra topological spaces," International Journal of Mathematical Analysis, vol. 6, no. 1, pp. 1-10, 2012.

[25] A. Kandil, O. Tantawy, S. A. El-Sheikh, and M. Hosny, "IPseparation axioms in ideal bitopological ordered spaces II," Sohag Journal of Mathematics, vol. 24, no. 2, pp. 279-285, 2016.
[26] T. M. Al-shami and M. E. El-Shafei, "On supra soft topological ordered spaces," Arab Journal of Basic and Applied Sciences, vol. 26, no. 1, pp. 433-445, 2019.

[27] A. M. Kozae, M. Shokry, and M. Zidan, "Supra topologies for digital plane," AASCIT Communications, vol. 3, no. 1, pp. 1$10,2016$.

[28] A. Alpers, "Digital topology: regular sets and root images of the cross median filter," Journal of Mathematical Imaging and Vision, vol. 17, no. 1, pp. 7-14, 2002.

[29] A. Mhemdi and T. M. Al-shami, "Functionally separation axioms on general topology," Journal of Mathematics, vol. 2021, Article ID 5590047, 5 pages, 2021. 